\title{
Collective dynamics support group drumming, reduce variability, and stabilize tempo drift
}

Dobromir Dotov $^{1,2}$, Lana Delasanta ${ }^{3,4}$, Daniel J Cameron ${ }^{1,2}$, Ed Large ${ }^{4,5,6}$, \& Laurel J Trainor ${ }^{1,2,7}$

${ }^{1}$ LIVELab, McMaster University, Ontario, Canada

2 Psychology, Neuroscience and Behaviour, McMaster University, Ontario, Canada

${ }^{3}$ Center for the Ecological Study of Perception and Action, University of Connecticut, Storrs, CT, USA

${ }^{4}$ Department of Psychological Sciences, University of Connecticut, Storrs, CT, USA

${ }^{5}$ Department of Physics, University of Connecticut, Storrs, CT, USA

${ }^{6}$ CT Institute for Brain and Cognitive Sciences, University of Connecticut, Storrs, CT, USA

${ }^{7}$ Rotman Research Institute, Toronto, Canada

* Corresponding author

dotovd@mcmaster.ca

Present address:

McMaster University

1280 Main St. West

Hamilton, ON L8S4K1 


\title{
COLLECTIVE DYNAMICS IN GROUP DRUMMING 2
}

\begin{abstract}
Humans are social animals who engage in a variety of collective activities requiring coordinated action. Among these, music is a defining and ancient aspect of human sociality. Social interaction has largely been studied in dyadic contexts. The presence of multiple agents engaged in the same task space creates different constraints and possibilities that have been studied more extensively in nonhuman animal behaviour. We addressed whether collective dynamics play a role in human circle drumming. The task was to synchronize in a group with an initial reference pattern and then maintain synchronization after it was muted. We varied the number of drummers, from solo to dyad, quartet, and octet. The observed lower variability, lack of speeding up, smoother individual dynamics, and leader-less inter-personal coordination indicated that stability increased as group size increased, a sort of temporal wisdom of crowds. We propose a hybrid continuous-discrete Kuramoto model for emergent group synchronization with pulse-based coupling that exhibits a mean field positive feedback loop. This research has theoretical implications about collective intentionality and social cognition.
\end{abstract}

Keywords: collective behavior, group dynamics, joint action, drumming, rhythm, network analysis 


\section{Collective dynamics support group drumming, reduce variability, and stabilize tempo drift}

\section{Introduction}

Humans are social animals who engage in a variety of collective activities requiring coordinated joint action. Collective goals can be achieved through spontaneously distributed workloads among group members, such that the emerging collective dynamics confer benefits to performance not available to individuals - the "wisdom of crowds" (Galton, 1907; Surowiecki, 2005).

Principles of collective dynamics explain the adaptive value of collective behavior in some species (Couzin, 2018). For example, collective dynamics can overcome the limitations of individuals' knowledge and ability to communicate (Goldstone \& Roberts, 2006) by integrating information quickly during group decision making (Ispolatov, 2015; Miller, Garnier, Hartnett, \& Couzin, 2013; Rosenthal, Twomey, Hartnett, Wu, \& Couzin, 2015). Large coherent swarming and flocking in groups arise from short-range interactions among proximal individuals in non-human animals including primates (Farine, Strandburg-Peshkin, Couzin, Berger-Wolf, \& Crofoot, 2017), insects (Jacobs et al., 2007), fish, and birds (Miller et al., 2013; Parrish, Viscido, \& Grünbaum, 2002). As these existing dynamics have been demonstrated in numerous species, theoretical models have been developed to provide additional quantitative explanatory support. In spatial tasks such as navigation, aggregate group behavior, formalized as a mean field, can serve to stabilize feedback to individuals by virtue of being a group average (Berdahl et al., 2018; Sumpter, 2006; Torney, Neufeld, \& Couzin, 2009). In the present paper, we test whether mean field behaviour accounts for stability of temporal group dynamics in humans during a drumming task.

Collective dynamics are observable in animal as well as in human group behaviour. Crowds of walking individuals achieve globally coherent states based on local inter-individual interactions (Rio, Dachner, \& Warren, 2018; Warren, 2018). In audiences, individual-group interactions and social contagion govern the spontaneous onset and offset of applause (Mann, Faria, Sumpter, \& Krause, 2013). Collective dynamics can also play a constitutive role in sports by permitting advantages not available to individuals alone (Vilar, Araújo, Davids, \& Bar-Yam, 2013). Evidence of collective dynamics is seen even in the minimal group, a dyad: Spontaneous synchronization between two individuals emerges from constraints such as weak coupling (Ouiller, de Guzman, Jantzen, Lagarde, \& Kelso, 2008; Schmidt, Carello, \& Turvey, 1990; Schmidt \& O’Brien, 1997).

Synchronized group action is an essential element of musicmaking, a defining social behaviour of human interaction (Honing, ten Cate, Peretz, \& Trehub, 2015; Patel \& Iversen, 2014; Salimpoor, 
Benovoy, Larcher, Dagher, \& Zatorre, 2011; Savage, Brown, Sakai, \& Currie, 2015; Trainor, 2015).The archaeological evidence of musical instruments goes back 30,000 years, and singing and drumming are thought to be even older (Conard, Malina, \& Münzel, 2009). The evolutionary origins of musical rhythmic actions may relate to social motor behaviors in non-human species, such as the synchronization and desynchronization of vocalizations between individuals in group chorusing, arising from pressures to either collaborate or compete (Gamba et al., 2016; Greenfield, Marin-Cudraz, \& Party, 2017; Ravignani, Bowling, \& Fitch, 2014; Ravignani, Verga, \& Greenfield, 2019).

Fundamental aspects of music are present in humans from early stages in development: infants show early musical preferences, social-emotional responses to music, and rate-sensitive motoric responses to musical rhythm (Cirelli, Einarson, \& Trainor, 2014; Cirelli, Trehub, \& Trainor, 2018; Trainor \& MarshRollo, 2019; Zentner \& Eerola, 2010). Although musical behaviour is found in individuals alone, it occurs mainly in groups ranging from duets to hundreds of participants. Yet, the role of collective dynamics, especially in groups larger than dyads, has largely remained untested. Here we consider a musical task in which timing consistency and synchrony is crucial. We investigate in what ways the group average performs better than the individuals, a temporal version of the wisdom of the crowd phenomenon.

Accounts of group behaviour often extrapolate theory developed for individual behavior and dyadic interaction. The idea that the brain is a prediction machine for motor control with respect to a changing and uncertain environment can be repurposed for dyadic interaction as two mutually predictive brains (Friston \& Frith, 2015; Wolpert, Doya, \& Kawato, 2003). In this context, the control of one's own timing and the prediction of the timing of the partner in the dyad are two separate processes (Heggli, Cabral, Konvalinka, Vuust, \& Kringelbach, 2019; Heggli, Konvalinka, Kringelbach, \& Vuust, 2021; Keller, Novembre, \& Hove, 2014; van der Steen \& Keller, 2013). Cognitive processes such as mutual prediction, however, may not necessarily provide an adequate foundation for explaining behaviour in larger groups. Increased group size challenges mutual prediction as every individual would have to predict every other individual and potentially factor in second-order predictions. Furthermore, the auditory information available to an individual within a group changes as group size increases. A listener is likely to perceive an entire choir as one coherent sound rather than perceive each and every singer's voice within that choir.

There are also models of coordination where prediction is not a separate process but is implicit in the emergent synchronization of coupled individual oscillatory dynamics (Alderisio, Bardy, \& di 
Bernardo, 2015; Ouiller et al., 2008; Schmidt et al., 1990; Schmidt \& O’Brien, 1997). In short, the one approach posits prediction for synchronization, the other, synchronization for prediction. An important benefit of the latter is that it naturally scales up to explain coordination in larger groups, converging on the same formalisms used for collective behaviour in animals (Kelso, 2021; Zhang, Beetle, Kelso, \& Tognoli, 2019). Here we consider such a system of coupled oscillators to account for variability in a group of drumming individuals.

Group music making constitutes an ecologically valid and convenient paradigm for studying group action and collective experience in the laboratory. We used a timing task performed by groups of different sizes. Larger group dynamics are less studied because measuring highly precise timing while collecting group data is difficult for both logistical and methodological reasons (cf, Alderisio et al., 2015; Chang, Kragness, Livingstone, Bosnyak, \& Trainor, 2019; Chang, Livingstone, Bosnyak, \& Trainor, 2017; Chauvigné, Walton, Richardson, \& Brown, 2019; Shahal et al., 2020). Participants completed a group synchronization-continuation task (SCT) (Figure 1A). It required them to drum in synchrony with an isochronous auditory stimulus and continue drumming at the same rate after the stimulus stopped, while we collected the onset times of each drum hit (Figure 1B). Stimulus tempo was varied across trials. We tested groups of 2 (dyads), 4 (quartets), and 8 (octets) participants (Figure 1C). The task tested participants' ability to synchronize to an external reference as well as to other participants, while also minimizing temporal variability and maintaining the initial stimulus rate. This ensemble drumming was also compared against the solo condition. Specifically, participants tested in duets or quartets completed both solo and ensemble conditions. The octet group did not complete the solo conditions for logistical reasons, but they instead completed a control condition in which the synchronization phase continued for the whole trial (i.e., there was no continuation phase) as well as trials using a more complex and musically realistic rhythm. Analyses of the more complex rhythm will be reported separately.

Inspired by theoretical models from the animal literature (Sumpter, 2006), we assumed that individual interactions average to overall group behavior, a mean field, which provides feedback to individual group members. Our main hypothesis was that the influence of the mean field increases with group size, which exercises a stabilizing effect. We expected that larger groups would exhibit lower variability as measured using the coefficient of variation of inter-onset intervals. After analyzing the recorded drumming behaviour, we formalized this effect using an adapted Kuramoto model of group synchronization that replaced continuous feedback with pulse-based feedback and added a stochastic 
COLLECTIVE DYNAMICS IN GROUP DRUMMING 6

term. In our model, individual group members with different intrinsic frequencies became dependent on the ensemble-feedback from the average of all other individuals, akin to a mean field. A key prediction was that the relative influence of the stabilizing feedback would increase with increasing ensemble size. It is also important to note that the model only involved mutual dynamic interaction without a separate process dedicated to prediction.

\section{Method}

\subsection{Participants}

A total of 102 university students participated in the duet ( $n=11$ sessions; 22 participants), quartet ( $\mathrm{n}=8$ sessions; 32 participants), and octet ( $\mathrm{n}=6$ sessions; 48 participants) groups. In addition, 88 highschool students participated in the octet condition $(n=11$ sessions $)$. Participants were either recruited from the department's participant pool with course credit or from local high schools as part of science field trips to the university.

\subsection{Apparatus}

In ensemble playing conditions, participants were seated in chairs facing each other, equally spaced in a linear, square, or octagon arrangement, with diagonal distances of three meters in the duet and quartet groups and six meters in octet group. Ensemble playing conditions were performed in a large, tall-ceiling dance studio, part of the LIVELab, a laboratory dedicated to auditory and movement neuroscience ${ }^{1}$. In the duet and quartet groups, solo trials were recorded with individual participants immediately before or after the ensemble sessions in an adjacent sound-proof room.

In the duet and quartet groups, participants used drum sticks on plastic buckets that were dampened and placed upside-down. These instruments were used by a group drumming workshop instructor in unrelated sessions and are of the type seen in street-style group drumming observed in the community. Piezoelectric sensor elements were taped on the inner side of the attack surface of each bucket. The analogue signal was recorded on a computer at $48 \mathrm{kHz}$ via a multi-channel studio-grade analogue-digital interface (Focusrite RedNet 4) and the digital audio production software Reaper (Cockos Incorporated, Rosendale, NY). This setup provided for a clean signal with minimal noise and a sharp, high-amplitude attack that was digitized into onset times in Matlab using a custom algorithm with thresholding and rejection of rebounds under $150 \mathrm{~ms}$. 
A

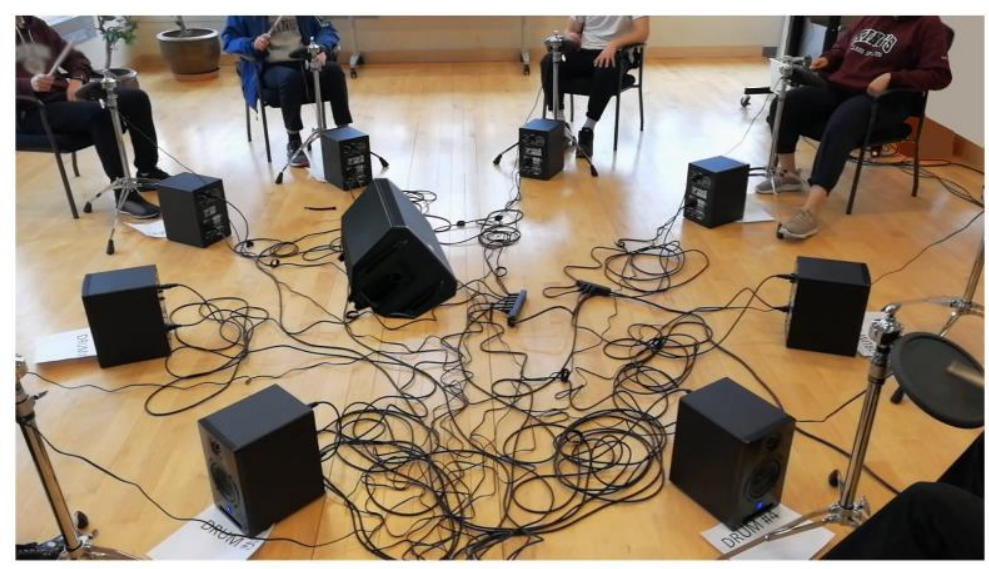

B

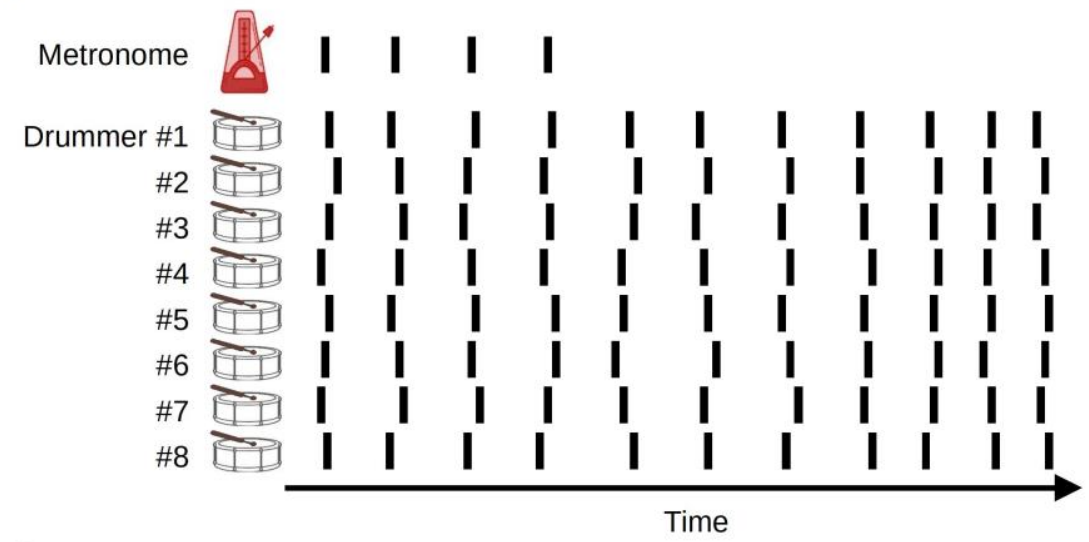

C
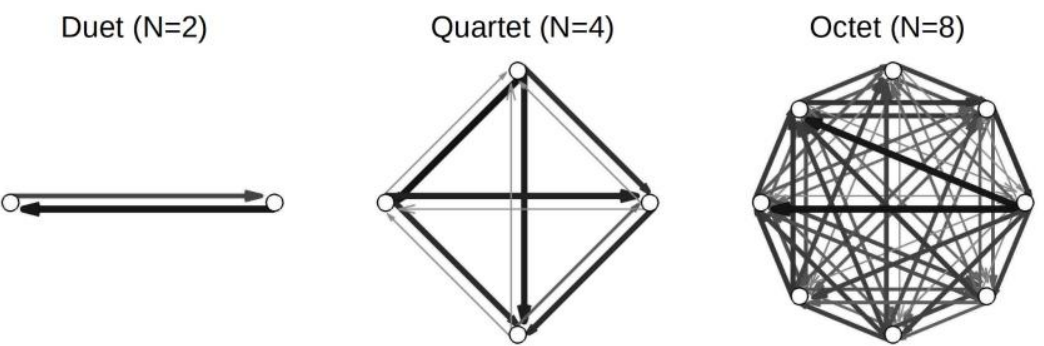

Figure 1. Experiment setup. (A) In ensemble condition, drummers faced each other in a circle. (B) The main task was synchronization-continuation where participants were paced initially by an auditory stimulus and then had to maintain the rhythm, tempo, and synchronization among each other. The inter-onset intervals between drum hits, shown schematically as vertical lines, were used to obtain individual-level measures variability and speeding up. Cross-correlation and transfer entropy were used as pair-level measures of synchronization and interaction. (C) Transfer entropies, color and width-coded, from three sample trials from different groups.

Network analysis was applied to these graphs to obtain group-level characterization. 
COLLECTIVE DYNAMICS IN GROUP DRUMMING 8

The octet group used drum pads from an electronic drum set. Eight identical drum pads (Yamaha TP70 7.5” snare/tom) and eight independent powered studio reference monitors (Yorkville Sound YSM5) placed under each pad were all wired to the head of the kit (Yamaha DTX 920K) and connected to the computer, which stored drum hits as MIDI events. The pads were configured with linear amplitude response, and, on impact, each pad produced the same woodblock sound, with small variations in pitch among drums to make them distinct. The two setups are similar as they both involve a polymer surface, a drumstick, and a sensor on the surface to detect impact and record onset times.

The reference metronome that participants were asked to synchronize with was played over a dedicated speaker placed in the middle between participants. A custom patch for the sound synthesis and production software Pure Data (Puckette, 1996) ran on the computer to generate and time a sharp electronic kick drum sound, with a timbre very distinct from the individuals' drums $(600 \mathrm{~Hz}$ sine wave with $250 \mathrm{~ms}$ duration, $20 \mathrm{~ms}$ amplitude attack, and $230 \mathrm{~ms}$ decay). By rerouting a parallel copy of this stimulus, it was recorded as a channel in the same data collection stream as the participants' drum beats.

\subsection{Stimuli}

The stimulus tempo of the isochronous reference metronome varied between 50 and 240 beats per minute (bpm) depending on condition (see below). Trials were $90 \mathrm{~s}$ long and the synchronization phase always included 32 stimulus events. Thus, slower tempos had longer duration synchronization phases.

\subsection{Task}

Drumming is ecologically valid in terms of common musical practice, and tapping with sticks has been shown to improve timing relative to finger tapping (Madison, Karampela, Ullén, \& Holm, 2013; Manning, Harris, \& Schutz, 2017). The task on each trial was a synchronization-continuation task (SCT). During the synchronization phase, participants were presented with trials containing a reference isochronous metronome at a particular tempo and were asked, using drumsticks, to: (1) synchronize with the given reference metronome, (2) keep their own variability as low as possible, (3) keep their drumming rate as steady as possible, and (4) synchronize with the other drummers when in an ensemble condition. During the continuation phase, the reference metronome stopped, and participants were told to continue to keep their drumming rate as steady as possible while synchronizing with other drummers in an ensemble. Those in the octet group also completed trials in a synchronization-only 


\section{COLLECTIVE DYNAMICS IN GROUP DRUMMING}

control task in which the isochronous metronome played for the entire trial (i.e., there was no continuation phase).

\subsection{Design}

Participants were assigned to one of three group sizes: duet (2), quartet (4) or octet (8). Those in the duet and quartet groups completed the SCT task in both solo and ensemble conditions. Duet and quartet groups completed 6 trials at each of the following tempos: 50, 80, 120, 160, 200, 240 beats per minute (bpm) (or 1200, 750, 500, 375, 300, 250 ms onset-to-onset intervals between beats) in each of the solo and ensemble conditions, with the order of tempos randomized. For each set of participants, there was random assignment to either the solo or ensemble condition first. Trials lasted $90 \mathrm{~s}$ with a synchronization phase of 32 metronome beats.

For octet groups, there were three differences in the design. First, they did not complete solo trials because of time and space constraints. Second, the set of tempos was slightly different $(80,100,120$, 160, $200 \mathrm{bpm}$ or 750, 600, 500, 375, $300 \mathrm{~ms}$ intervals). Finally, an additional condition was included, consisting of a synchronization-only control task (i.e., with the reference metronome throughout).

We found that the very low and very high tempos led to poor performance, hence we eliminated extreme tempo trials and only analyzed the set of matching tempos across dyads, quartets, and octets, namely 80, 120, 160, and $200 \mathrm{bpm}$. For all groups, prior to completing the trials in each condition, participants engaged in practicing synchronized steady drumming without an initial metronome to set the tempo, so that participants could become familiar with the sticks and drums and playing together in an ensemble.

\subsection{Procedure}

After providing informed consent, filling out demographic and music background questionnaires, reading instructions, and observing a demonstration of the apparatus, participants were instructed to find a comfortable posture on their chair and adjust the drum position during practice. They were asked not to adjust these further during the trials. Participants were allowed to practice as needed to feel comfortable with the task. After each trial, the experimenter verified with the participants that they were ready to start drumming again before the next trial was initiated. The whole session from arrival to departure lasted about an hour. 
During the solo performance in the duet condition, one participant was moved to an adjacent sound-proof room while the second remained in the large dance studio. For the quartet condition, two of the participants were randomly assigned to arrive earlier and perform their solo trials before the group trials and the other two to perform their solo trials after the group trials.

\subsection{Analysis}

\subsubsection{Pre-processing of onset times}

Onset times were processed using custom Matlab scripts. In SCT trials, only data from the continuation phase was analyzed. In the synchronization-only control trials of the octet group, the full trial length was analyzed. The following steps were taken to remove artifacts and condition the data according to the requirements of the analyses used.

2.7.1.1 Signal. On a few occasions participants accidentally pulled the cable from the drum and disconnected or damaged the sensor. A total of fifteen bad channels (i.e., participants) were detected and removed from further analysis with a custom script using the onset variability, signal noise, and amplitude consistency. For further verification, we visually inspected the signals and onset times from all channels and trials.

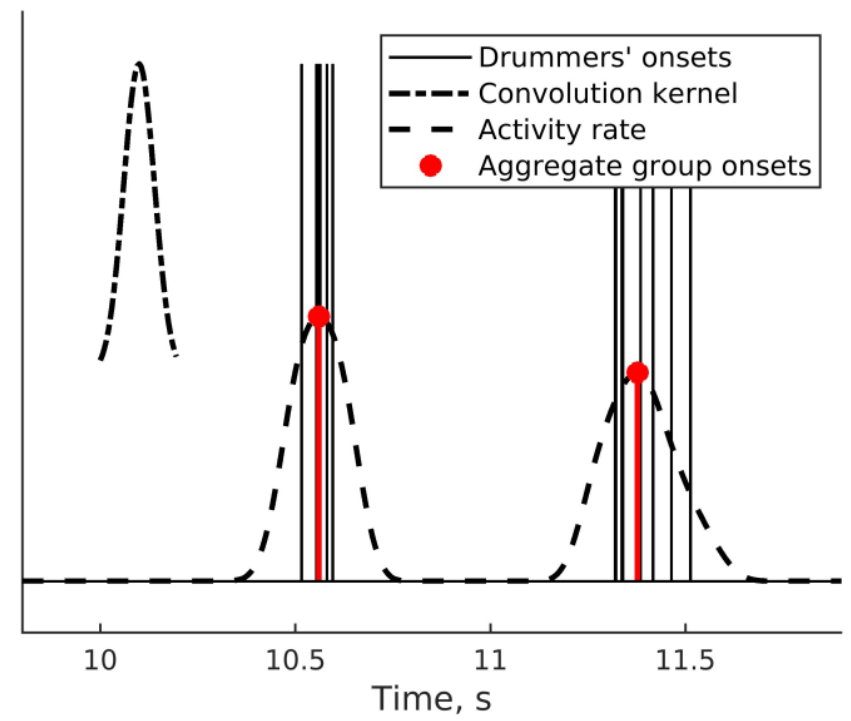

Figure 2. Group-aggregate onset times from convolving the drummers' onsets with a Gaussian kernel.

\subsubsection{Group-aggregate (mean field) measure for ensembles and pseudo-ensembles. For}

ensemble trials, we created a measure of the beat onset times of the group as a whole by constructing 


\section{COLLECTIVE DYNAMICS IN GROUP DRUMMING}

theoretical group-aggregate onsets in the center of clusters of individuals' onset times, $t_{n, g}$, where $\mathrm{n}$ is the beat onset number and g signifies it is the group-aggregate onset. Specifically, we: (1) convolved onset times with a Gaussian kernel $200 \mathrm{~ms}$ in width, (2) smoothed the resulting group activity rate with a moving average window of a quarter of the reference stimulus beat onset-to-onset time, and (3) took the peak locations for group-aggregate beat onset times (see Figure 2). This algorithm, typically used in neuroscience to extract a firing rate or population activity from the spike times of multiple recorded units (Dayan \& Abbott, 2001), provides a measure of central tendency of one or more events clustered in time. The method does not necessarily restrict the group-aggregate pattern to one average onset because if a single onset time is isolated away from other clusters, for example when a participant erroneously hits the drum while the other participants were silent, it will also be detected as a singleevent cluster. Such events of large individual deviations from the group were very rare in the present study and eliminated from analysis because, overall, participants performed the task consistently and in agreement with group members. Group-aggregate beat onset times were calculated for each trial in each group during ensemble playing conditions. In solo trials of dyads and quartets, participants could not see or hear each other. Still, we computed in the same way beat times of the pseudo-groupaggregate to obtain a baseline and verify that the procedure did not create spuriously periodic groupaggregates, see Supplementary Materials, Figure S1.

2.7.1.3 From beat onset times to IOIs, outlier removal, clustering. For each trial for each participant, the sequence of onset times $t_{n, i}$, where $i$ is participant and $n$ onset number, were differenced to produce a time-series of inter-onset-intervals, $\mathrm{IOI}_{n, i}=t_{n, i}-t_{n-1, i}$. Where appropriate for a more intuitive interpretation, we expressed these as tempos, $\mathrm{T}_{n, i}=60 / \mathrm{IOI}_{n, i}$. The same procedure was carried out to obtain the time-series of IOIs for the group-aggregate measure in ensemble conditions and the pseudo-aggregate measure in solo playing conditions.

If a participant skipped a beat on a certain occasion this would produce an outlier IOI twice as large as most others in the trial, thus biasing subsequent measures. IOIs exceeding $50 \%$ difference above or below the trial median were removed. The measures of individual variability and tempo trend (see below) were based on these IOIs.

2.7.1.4 Alignment of IOIs. Quantifying inter-personal coordinated tapping with the crosscorrelation requires pairing IOIs from all participants in a given trial. To this end, we used a custom algorithm to detect IOIs that were matching across all group members. To illustrate, if one group 


\section{COLLECTIVE DYNAMICS IN GROUP DRUMMING}

member skipped a beat, the produced IOI was about double that of the others and the corresponding IOIs for all group members were removed.

2.7.1.5 Pre-whitening. Cross-correlations are difficult to interpret in the presence of nonstationarities such as slow drift up and down of the IOI. We therefore applied a method for removing such non-stationarities by pre-whitening the IOIs (Dean \& Dunsmuir, 2016). Specifically, each timeseries of IOIs was filtered using an auto-regressive model with coefficients estimated with automatic model-order selection ('prewhiten' function from the TSA package for R).

\subsubsection{Performance measures and dynamics}

2.7.2.1 Variability and tempo trend. The tempo trend was defined as the linear trend of IOIs along successive beats in a trial, the slope $b$ in the regression equation $T_{n, i}=a+b n_{i}$, fitted separately in each trial for each individual. In ensemble conditions, we only kept the group-aggregate tempo trend because participants were well coordinated and they all played at essentially the same overall tempo, producing nearly identical $b$ 's.

After removing the overall group trend $b$, variabilities of individuals and group-aggregates were estimated separately using the coefficient of variation of the IOIs.

2.7.2.2 Auto-correlation. Individuals' and group-aggregate dynamics were analyzed using autocorrelation of IOIs.

\subsubsection{Inter-personal coordination}

2.7.3.1 Cross-correlation. The temporal pattern of co-variation among participants was analyzed using cross-correlation applied to the pre-whitened IOIs.

2.7.3.2 Transfer entropy. The bidirectional interaction between every pair of participants in a group was measured using transfer entropy (TE) (Schreiber, 2000). This is an information-theoretic measure of directed mutual information used for quantifying the effective connectivity in, for example, moving animals (Brown, Bossomaier, \& Barnett, 2020), sensori-motor and motor-motor interactions (Dotov \& Froese, 2018; Lungarella \& Sporns, 2006; Stoffregen, Villard, Kim, Ito, \& Bardy, 2009), and complex networks of neurons (Gourévitch \& Eggermont, 2007). Effective connectivity, sometimes also referred to as causality, is understood in the Wiener sense that having information about past source dynamics improves the prediction of future target dynamics (Wiener, 1956). We used a Matlab toolbox for TE (Ito et al., 2011) adapted for point processes such as neuronal firing times where the source $(J)$ and target $(I)$ dynamics are binary events separated by a delay $d$, 
$T E_{J \rightarrow I}(d)=\Sigma p\left(i_{t+1}, i_{t}, j_{t+1-d}\right) \log _{2} \frac{p\left(i_{t+1} \mid i_{t}, j_{t+1-d}\right)}{p\left(i_{t+1} \mid i_{t}\right)}$. The $t$ indices here are bins of time, set to $10 \mathrm{~ms}$. We took the maximum TE over a range of up to 100 delay steps (i.e., $1 \mathrm{~s}$ ). We performed the analysis either with or without removing the trend of decreasing intervals between time indices in a trial (i.e., trend for tempo to speed up), and found this did not change the pattern of results.

Arguably, TE has several benefits over comparable methods (Lungarella, Pegors, Bulwinkle, \& Sporns, 2005). We identified another reason for applying TE to the sequence of time onsets $t$ rather than what would be the first choice historically, namely TE or Granger causality among sequences of IOIs paired across participants. The latter scenario involves several steps of subtractive pre-processing consisting of removing outliers due to missed taps, pruning and aligning observations across participants, and filtering smooth trends that could be intrinsic to the performance, whereas applying a version of TE for point processes (sequences of onsets) avoids the steps which risk excessive data removal.

\subsubsection{Network analysis}

The measures in Section 2.7.2 are univariate and describe drumming performance of individuals. This individual-level analysis does not say much about how participants coordinate with each other to achieve group performance. Section 2.7.3 lists bi-variate measures typically used to analyze dyadic tapping and sensorimotor coordination problems. Yet, even such pair-level analysis is not sufficient in the group context where more than two participants coordinate simultaneously with each other. Hence, we obtained group-level measures by analyzing the network of effective connectivity among drummers (Newman, Barabási, \& Watts, 2011). Drummers were treated as nodes (or vertices) and TE values as the weights of links (or edges) of a directed graph.

2.7.4.1 Causal density and Mean node strength. Among the rich set of phenomena that potentially can be quantified in a large network, only a few measures are relevant in the present context because of the low number of nodes and the real-valued nature of the graph consisting of continuous connection weights rather than binary links. We defined causal density as the average of all edge weights (Seth, Barrett, \& Barnett, 2011; Seth \& Edelman, 2004). Mean node strength (Barrat, Barthélemy, PastorSatorras, \& Vespignani, 2004), obtained using the igraph package for R (Csardi \& Nepusz, 2006), was defined as the average across drummers of each drummer's total outbound connectivity. This is a version of the more popular measure of node degree which is used for binary graphs. 


\subsection{Statistical Analyses}

Linear mixed effects models (Bates, Mächler, Bolker, \& Walker, 2014; Singer \& Willett, 2003) were used instead of ANOVAs because of the complex, unbalanced design and missing observations. The Satterthwaite method was used to determine significant effects and interactions (Kuznetsova, Brockhoff, \& Christensen, 2017). By taking different subsets of the data and fitting separate models, we tested for: (1) effects of group size in SCT trials comparing dyads, quartets and octets, (2) differences between solo and ensemble playing conditions in dyads and quartets, (3) differences between SCT and synchronization-only in octets, and (4) differences between individual and groupaggregate performance. For brevity, only the selected model with best fit is reported in Results. The modeling procedure was incremental, starting with the minimal specification of a grand mean and iteratively including predictors and their interactions until the model became over-specified or too complex to converge, which tended to be the case when including tempo. Tempo will be treated in more detail in another paper focused on its theoretical significance.

\subsection{Theoretical account}

\subsubsection{The Kuramoto Model of emergent collective synchrony}

The Kuramoto (1975) dynamic system of coupled phase oscillators, Eq. 1, was conceptualized as a large population of oscillators with different natural frequencies capable of spontaneously locking to a common frequency.

$$
\dot{\theta}_{i}=\omega_{i}+\frac{K}{N} \sum_{j=1}^{N} \sin \left(\theta_{j}-\theta_{i}\right)
$$

Here $\theta_{i}$ is the phase of oscillator $i, \omega_{i}$ is its preferred frequency, i.e. how fast around the unit circle $\theta_{i}$ it likes to go, $K$ is coupling strength, and $N$ is the number of oscillators.

The model gives a concrete mathematical account of group synchronization as dependent on a mean field, referred to here as group-aggregate. The central feature of the model is that the feedback is positive: the amplitude of the mean field grows as a function of inter-individual synchronization and, reciprocally, the individual oscillators are affected more by the mean field if its amplitude is larger, see Figure 3. This can be seen by looking at the definition of the mean of phases, Eq. 2, and expressing Eq. 1 equivalently ${ }^{2}$ in terms of the coupling between individual oscillators and the mean field, Eq. 3, where

\footnotetext{
${ }^{2}$ The mean-field approximation in Eq. 2 is analytically exact in the limit of infinitely large groups (Strogatz, 2000).
} 


\section{COLLECTIVE DYNAMICS IN GROUP DRUMMING}

$\psi$ is the mean field phase and $r$ is the mean field coherence, also called order parameter (Kuramoto, 1975).

$$
\begin{aligned}
& r e^{i \Psi}=\frac{1}{N} \sum_{j=1}^{N} e^{i \theta_{j}} \\
& \dot{\theta}_{i}=\omega_{i}+r K \sin \left(\Psi-\theta_{i}\right)
\end{aligned}
$$

Such individual-collective positive feedback loops enable ant trails and other phenomena in swarming animals (Sumpter, 2006) as well as acoustic herding in chorusing animals (Ravignani et al., 2014). The system also serves as a model for neuronal collective synchronization (Breakspear, Heitmann, \& Daffertshofer, 2010; Frank, Daffertshofer, Peper, Beek, \& Haken, 2000; Noori et al., 2020). Theoretically, the principles embodied by this system should apply to group action in humans too (Zhang et al., 2019). It has found application in understanding inter-personal synchronization of dyads (Dotov et al., 2019; Heggli et al., 2019; Roman, Washburn, Large, Chafe, \& Fujioka, 2019) and larger groups (Alderisio et al., 2015). In the present context, the model predicts that ensembles will be more stable than individuals because of the feedback loop between the timing of individuals and the group-aggregate of individuals.

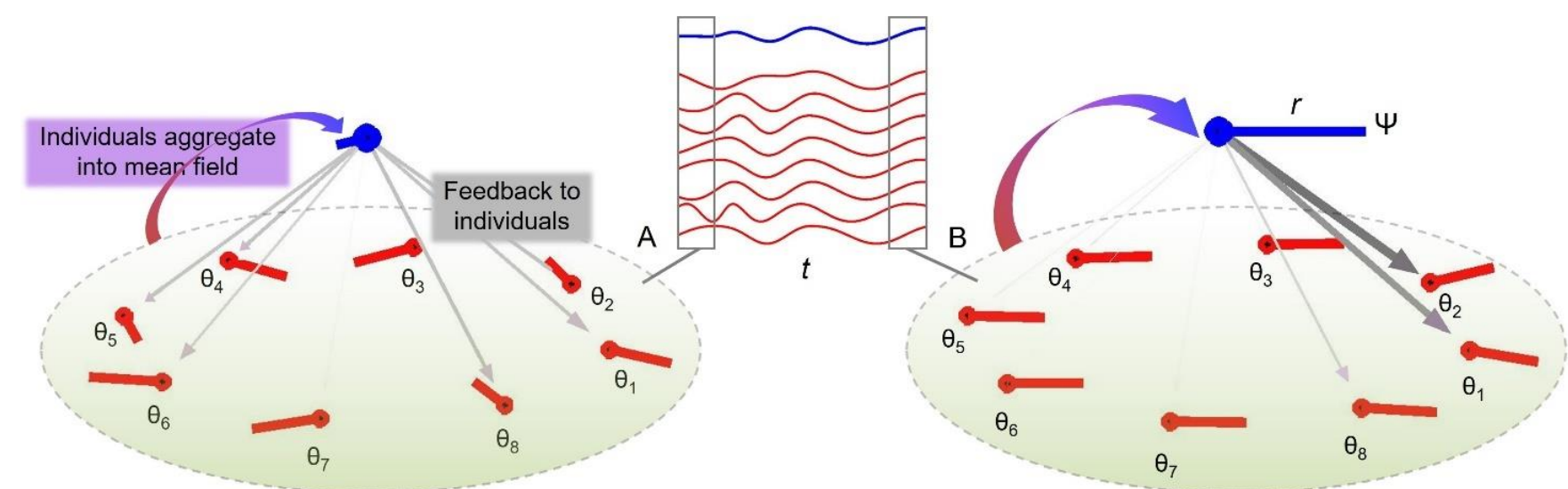

Figure 3. Group synchronization of eight oscillators with random initial conditions $(A)$ and coherent phases later in the trial (B). The middle inset shows individual trajectories (red lines) and the mean field (blue line) in time $(t)$. Averaging the phase oscillators $\theta$ (red lines) gives a so-called mean field (blue lines) with phase $\Psi$ and amplitude $r$. As the individual oscillators become more coherent from (A) to (B), $r$ increases which leads to stronger influence from $\Psi$ to diverging $\theta$ 's (weight of the downward vectors). 


\subsubsection{Hybrid continuous-discrete model of group synchronization}

The original Kuramoto model involves continuous dynamics and continuous coupling with constant gain and no delay. In a drumming task participants are coupled by way of discrete acoustic onset times. Here we propose a hybrid continuous-discrete system of oscillators with event-based feedback updated once per cycle, Eq. 4. It utilizes a pulse function, Eq. 5, in the form of a gamma distribution with parameters $a=1.25$ and $b=0.02$, shaped like the acoustic envelope of a drum hit. This means that a pulse is emitted at a discrete moment in time when an oscillator's phase angle completes one loop. Furthermore, we posit additive frequency variability with a Gaussian distribution $\mathcal{N}(0, \sigma)$.

$$
\begin{aligned}
& \dot{\theta}=\omega_{i}+\frac{K}{N} \sum_{j=1}^{N} P_{j}(t) \sin \theta_{i}+\mathcal{N}(0, \sigma), \quad i=1, \ldots, N \\
& P_{j}(t)=f(t \mid a, b)=\frac{1}{b^{a} \Gamma(a)} t^{a-1} e^{\frac{-t}{b}}
\end{aligned}
$$

Importantly, just like the Kuramoto system, this model involves intrinsic dynamics $\left(\omega_{i}\right)$ and interaction dynamics (the whole coupling term) but no separate internal terms dedicated to predicting partners' dynamics.

We simulated 100 runs per group size $N=\{2,4,8\}$ where each run corresponded to a trial with duration of 30 seconds. Coupling strength was $K=0$ in solo and $K=5$ in ensemble conditions. The noise term had a mean of zero and variability $\sigma=2$. These parameters reproduced our behavioural data qualitatively. We centered the uniform distribution of intrinsic frequencies $\omega_{i}$ at $2 \mathrm{~Hz}$ (range of .4 Hz) by considering that comfortable finger tapping is in the range 1.5-2 Hz (McAuley et al., 2006), tapping is optimally precise in the range $0.8-2.5 \mathrm{~Hz}$ (Moelants, 2002), and walking on average is at $2 \mathrm{~Hz}$ (MacDougall \& Moore, 2005). Performance variability was measured in terms of the coefficient of variation of cycle durations, treating zero-phase crossings as onset times.

\section{Results}

\subsection{Performance}

\subsubsection{Variability.}

3.1.1.1. Does individual variability increase in ensemble compared to solo conditions? To answer this question, we fitted a model including only individuals' data in dyads and quartets. (Octets did not complete solo conditions.) As Figure 4A shows, individuals' variability increased when playing in an ensemble relative to playing solo $[\beta=.009, \mathrm{SE}=.001, t=6.65]$. 

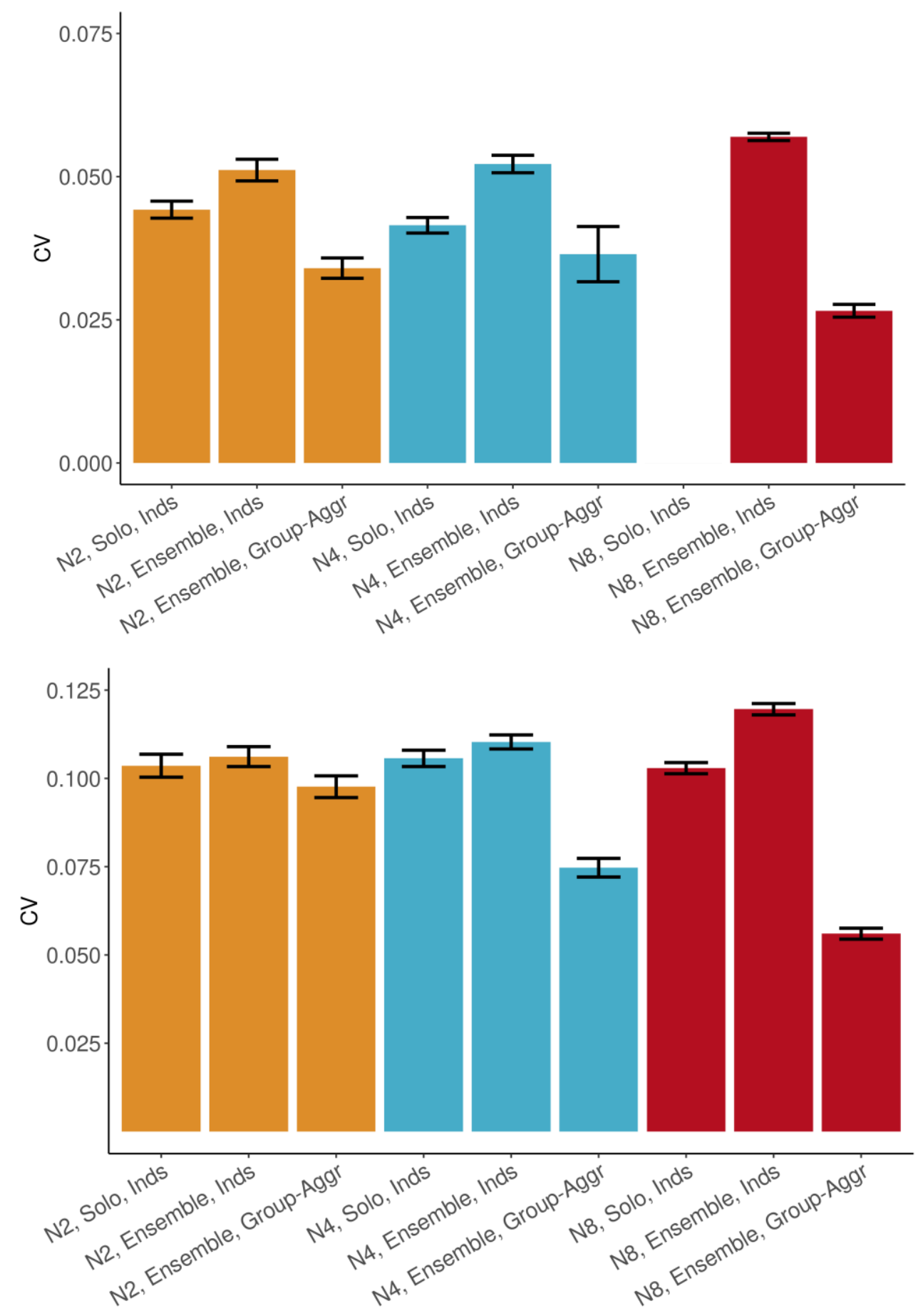

Figure 4. Task performance measured in terms of mean (SE) coefficient of variation of IOls. Top Graph. SCT drumming trials (no data collected in N8 Solo Condition). Bottom Graph. Stimulation data. N2 = dyad; N4 = quartet; N8 = octet; Inds = individual participants; Solo = solo condition; Ensemble = ensemble condition; Group-Aggr = group-aggregate. 


\subsubsection{In the context of true ensemble playing, how does variability of the individuals and group-} aggregate change across dyads, quartets, and octets? To specifically test our main hypothesis, we fitted a model to continuation (SCT) trials from all three group sizes in the ensemble playing condition. We found that individual variability increased with group size $[\beta=.001, \mathrm{SE}=.0005, t=2.20]$, groupaggregate variability was lower than individual variability $[\beta=-.009, \mathrm{SE}=.003, t=-3.29]$, and the difference between individual and group-aggregate variability increased for larger group sizes $[\beta=-.0025, \mathrm{SE}=.0005, t=-5.19]$, see Figure 4B.

3.1.1.3. How does variability compare between continuation and synchronization? The octet group completed trials in conditions of both SCT (i.e., after the offset of the reference metronome) and synchronization-only (with a constant reference metronome). Surprisingly, there were no differences between these conditions in either in individual $[t<1]$ or in group-aggregate variability $[\beta=-.003$, $\mathrm{SE}=.002, t=-1.55]$.

\subsubsection{Speeding up.}

\subsubsection{Do individuals speed up more when playing solo than in dyad or quartet ensembles?} Speeding up was defined as the linear increase in tempo (i.e., decrease of IOIs) over the course of a trial. As expected, individuals sped up even when playing solo $[\beta=.019, \mathrm{SE}=.007, t=2.82]$ and greater speeding up was observed in ensemble than in solo conditions $[\beta=.022, \mathrm{SE}=.006, t=4.02]$. The effect of group size was not significant $[t<1]$.

3.1.2.2. Do dyad, quartet, and octet ensembles speed up to different extents? To test the effect of group size across the duet, quartet, and octet conditions, a second model was fitted with group size as a continuous predictor, including only trials from the SCT ensemble playing. As evidenced by Figure 5, there was a trend for speeding up to decrease as group size increased, which was not significant $[t<1]$. However, the interaction between speeding up and tempo was significant $[\beta=-.000037, \mathrm{SE}=.000013$, $t=-2.76]$, reflecting that at higher tempos larger ensembles sped up less than smaller ensembles, see Supplementary Materials, Figure S2B. 


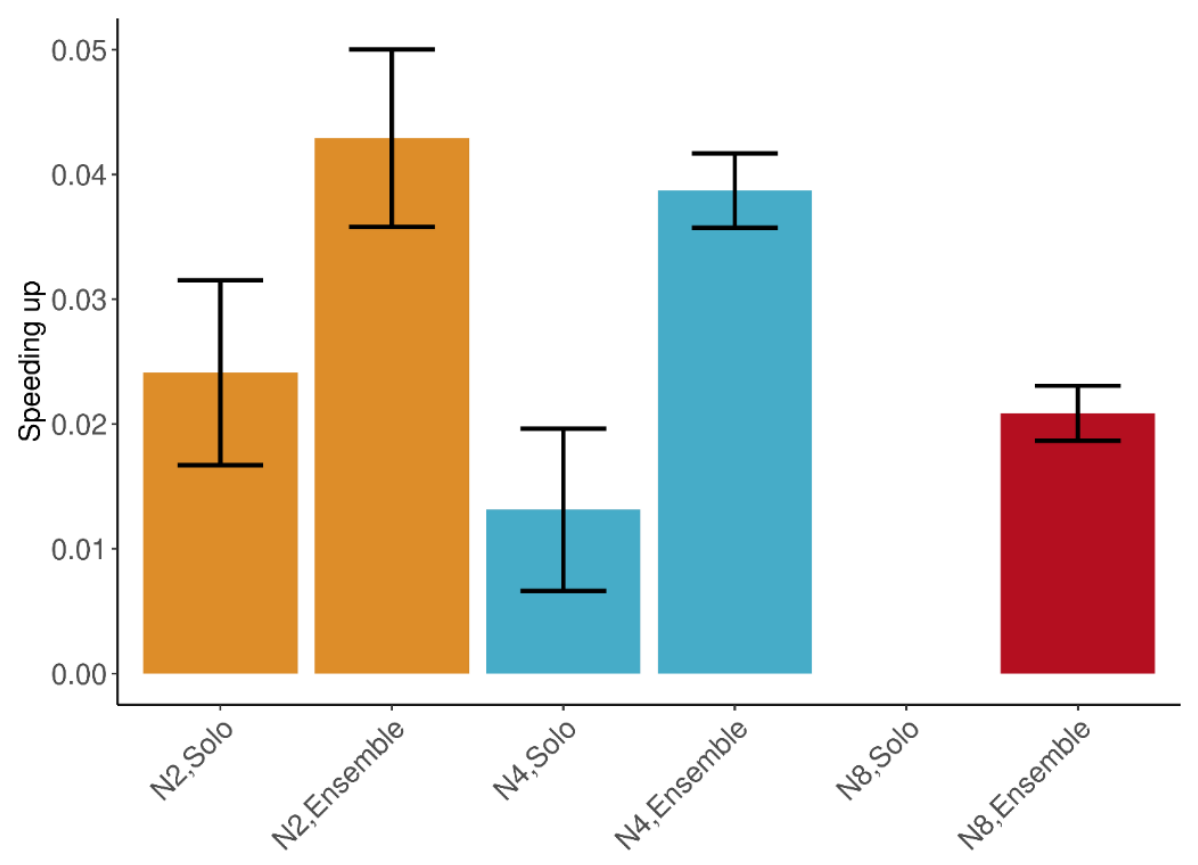

Figure 5. Mean (SE) speeding up defined as the linear slope of tempo over time, computed from the IOls. N2 = dyad; N4 = quartet; N8 = octet. (No data collected in N8 Solo condition.)

\subsection{Dynamics and coordination in ensemble, SCT conditions}

\subsubsection{Individual and group-aggregate dynamics (autocorrelations).}

3.2.1.1. Individuals. Figure 6A shows that individuals' autocorrelations at lag1 were negative across all group sizes and tempos. This is typical for the alternating long-short IOIs seen in synchronization tasks, reflecting alternating fast-slow synchronization errors when adapting to the previous interval. There was also a trend across all group sizes and tempi for positive peaks at even lags $(2,4,6$ and 8$)$ and negative peaks at odd lags (3, 5, and 7), see Supplementary Materials, Figure S3. However, this general pattern became smoother with increasing group size. To test this statistically, in each trial we took the average absolute difference between successive lags, thus measuring the average range of the autocorrelation function up to lag 8, and applied the same linear modeling approach as in Section 3.1. The effect of group size was significant [ $\beta=-.0049, \mathrm{SE}=.0021, t=-2.32]$.

There was also a decrease of range with increasing tempo $[\beta=-.00038, \mathrm{SE}=.0001, t=-4.02]$. Additionally, we verified the signs, relative magnitudes, and the effects of group size and tempo on the autocorrelations by fitting separate linear models for each lag, see Supplementary Materials, Table 1a. 


\section{COLLECTIVE DYNAMICS IN GROUP DRUMMING 20}

3.2.1.1. Group-aggregate. Interestingly, group-aggregate IOIs revealed the same overall pattern of results, see Figure 6B and Supplementary Materials, Table 1b, even though by definition the groupaggregate timing was smoother and less variable (Section 3.1.1). There was a trend across all group sizes and tempi for relative positive peaks at even lags 2, 4, 6 and 8, and relative negative peaks at odd numbered lags $1,3,5$, and 7 . The similarity of the dynamics of individuals and the group-aggregate is not trivial because it implies that even groups of up to eight participants spontaneously acquire the alternating long-short interval dynamics characteristic of when individual participants synchronize with a stimulus. There were also effects of tempo, see Supplementary Materials, Table $1 \mathrm{~b}$.
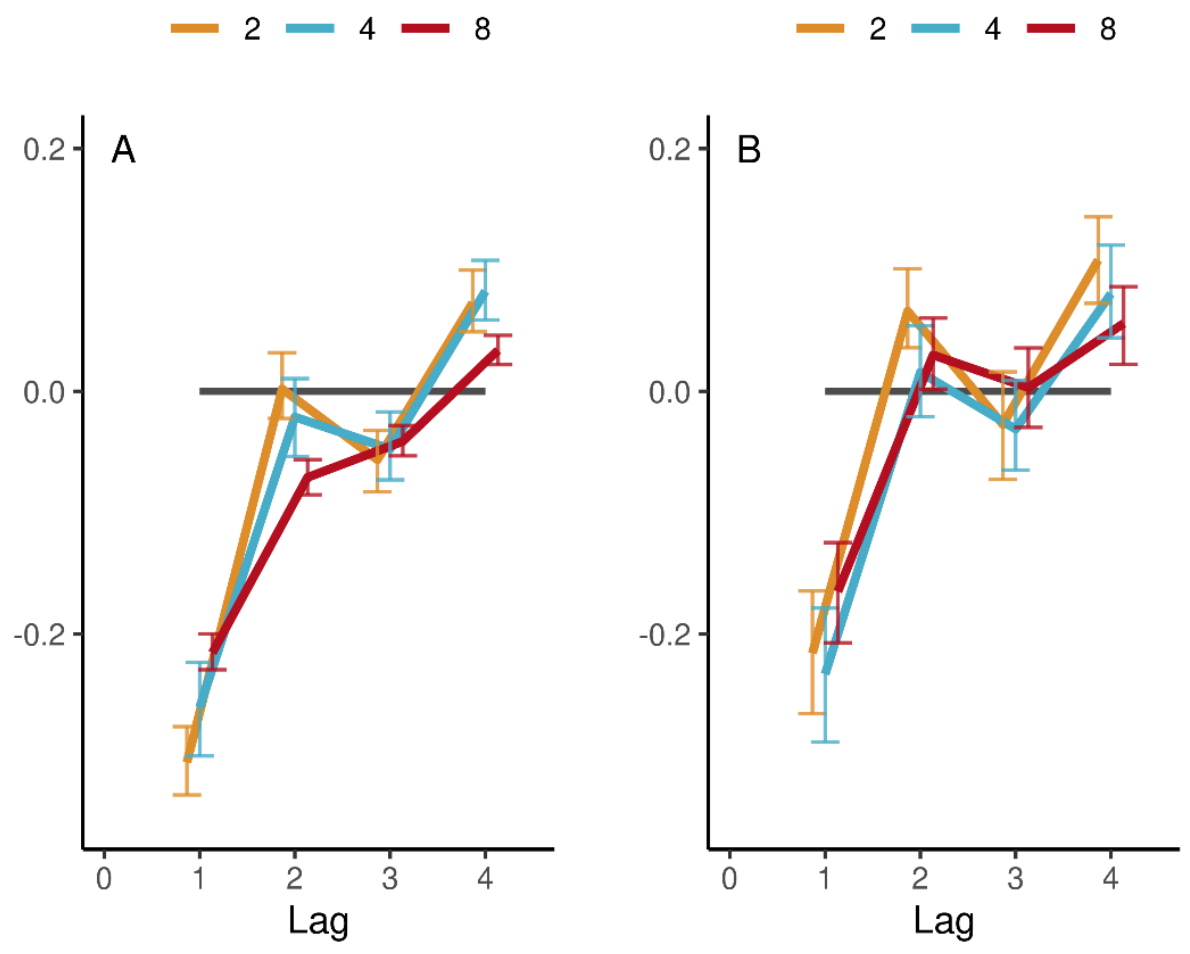

Figure 6. (A) Autocorrelations of individual IOls in the condition of ensemble SCT drumming, averaged (SE) across participants' trials and tempos, separately per group size (color-coded). (B) Same for group-aggregate IOls. 


\section{COLLECTIVE DYNAMICS IN GROUP DRUMMING}

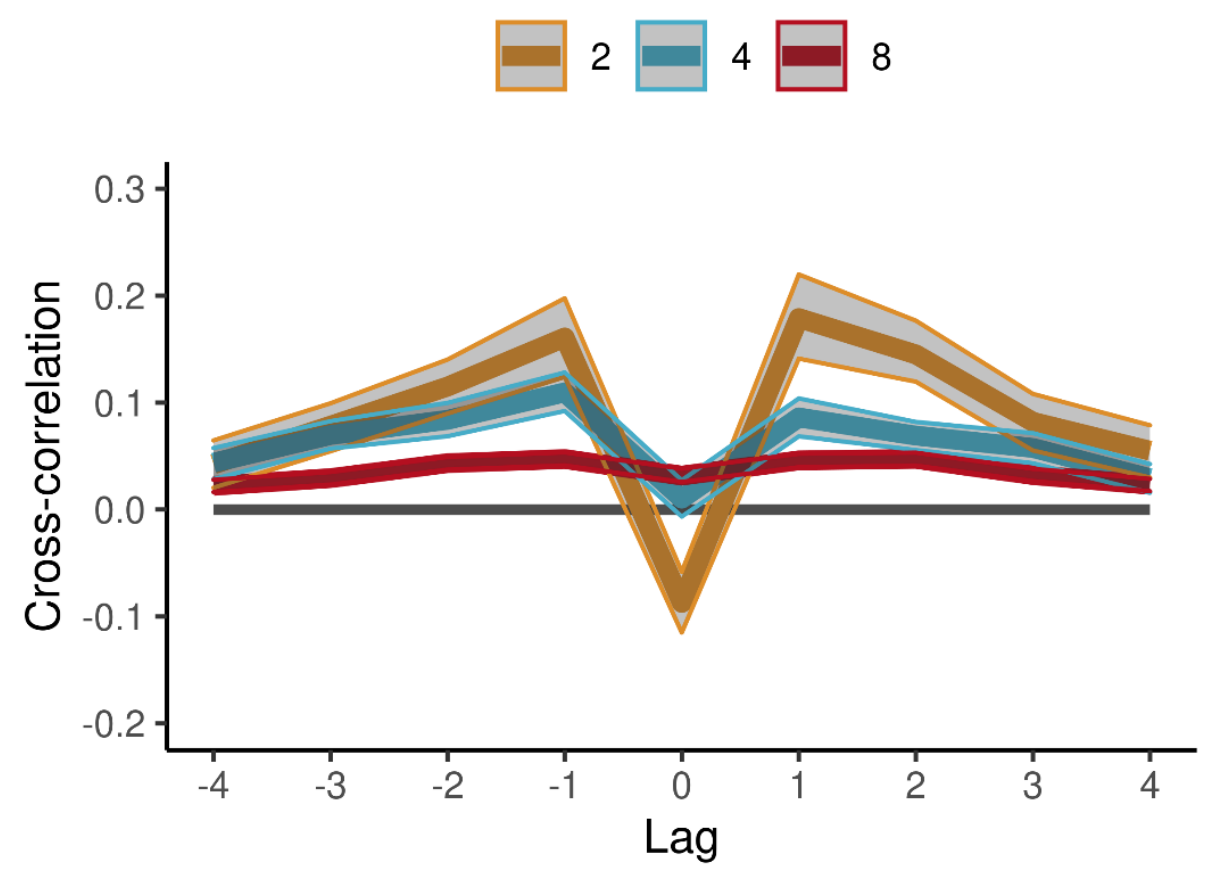

Figure 7. Cross-correlations in the SCT task in ensemble conditions. Averages (SE) across participant pairs and tempos are shown separately per group size (color-coded lines). IOls were aligned across participants and pre-whitened by filtering with an autoregressive model.

3.2.2 Inter-personal coordination (cross-correlations). Cross-correlation applied to the preprocessed IOIs assessed inter-personal coordination between pairs of participants drumming together in the continuation phase of ensemble performances. Specifically, outlier IOIs were removed, and the remaining IOIs were matched across participants by proximity and then pre-whitened (see Sections 2.7.1.3-5). We analyzed lags in the range $0-4$ because cross-correlation coefficients tended to be symmetric between positive and negative lags, see Figure 7 and Supplementary Materials, Figure S4. As expected, cross-correlations at lag0 were negative in duets $[\beta=-.041, \mathrm{SE}=.011, t=-3.60]$, consistent with the pattern of results for autocorrelations, and inter-personal coordination dynamics were smoother in larger group sizes. Furthermore, lag0 cross-correlations became less negative with increasing group size $[\beta=.012, \mathrm{SE}=.002, t=5.93]$. $\operatorname{Lag} 1$ cross-correlations were positive $[\beta=.14$, $\mathrm{SE}=.012, t=11.80]$ but their magnitude decreased with group size $[\beta=-.016, \mathrm{SE}=.002, t=-7.14]$. There were also effects of tempo, see Supplementary Materials, Figure S4. 


\subsection{Group dynamics}

3.3.1 Network analysis. We used network analysis in the continuation phase of trials of ensemble playing to describe group coordination at an even higher level of organization than interpersonal coordination. First, we obtained the directed graphs of functional connectivity among participant drummers. In each trial, the graph consisted of the real-valued directed links between pairs of drummers estimated by way of the transfer entropy (TE), see Figure 1C. Then, network properties of these graphs were computed, see Section 2.7.4. Mean node strength increased with group size $[\beta=.0032, \mathrm{SE}=.00032, t=10.12]$ and was not affected by tempo ${ }^{3}[t<1]$, Figure $8 \mathrm{~A}$. There was a marginally significant effect of group size on causal density $[\beta=.0013, \mathrm{SE}=.00064, t=2.01]$. Causal density increased with tempo $[\beta=.0179, \mathrm{SE}=.0039, t=4.51]$, but less so for larger groups $[\beta=-.00197$, $\mathrm{SE}=.00067, t=-2.94]$, the combined effect being that causal density was lower for larger groups, see

Figure 8B. Comparing between SCT trials and synchronization-only trials in the octet group found no difference for causal density $[t<1]$ or mean node strength $[t<1]$, suggesting group dynamics were similar regardless of whether a pacing stimulus was present or not.
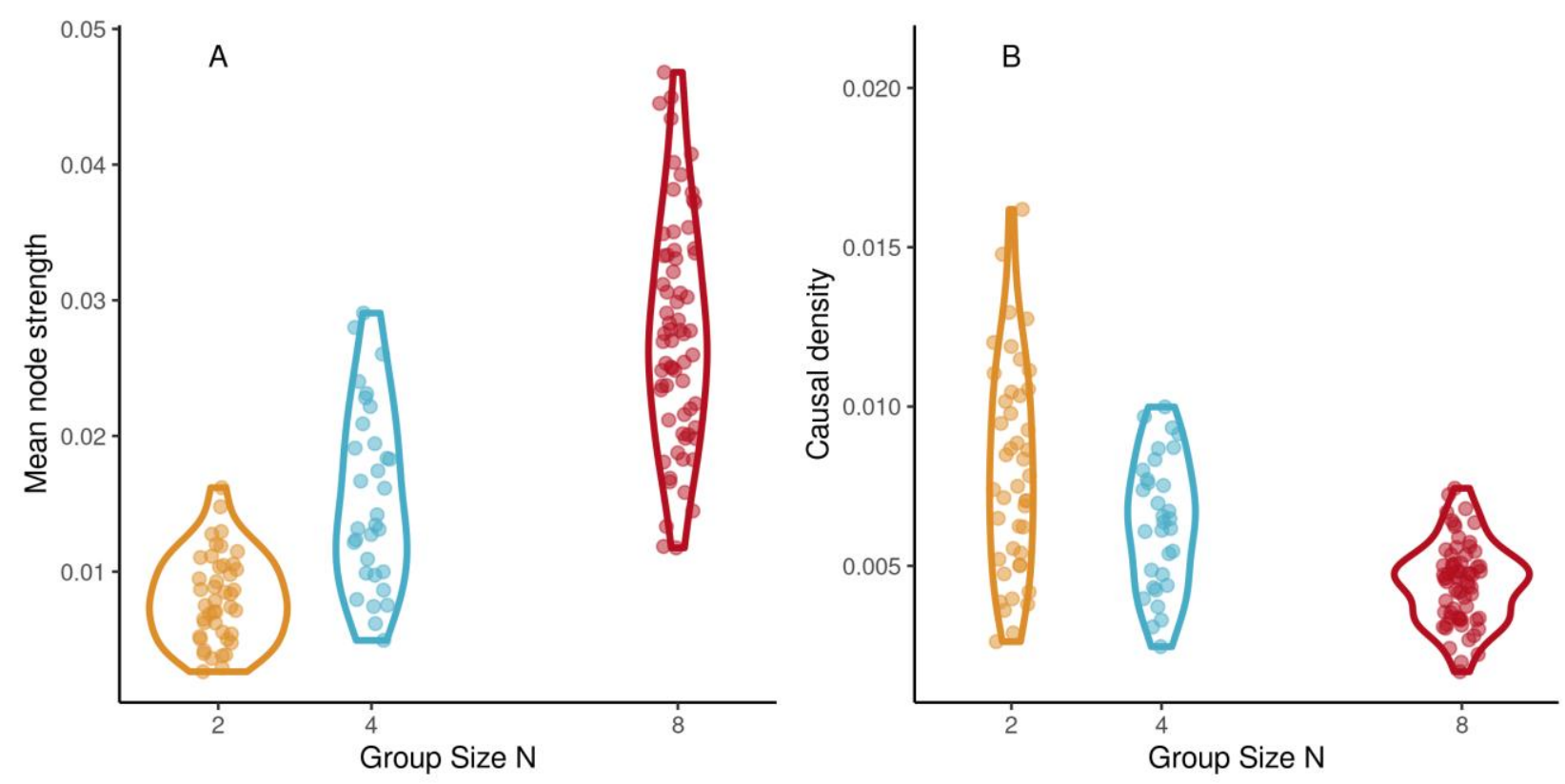

Figure 8. Network dynamics. A. Mean node strength increases with group size in the continuation phase of SCT, ensemble drumming trials. B. Causal density decreases with group size. (Abscissa jittered for visibility.)

\footnotetext{
${ }^{3}$ For better model conditioning, tempos values were reduced and compressed by transforming them with a logistic function.
} 
3.3.1 Network analysis and task performance. The relation between group dynamics and group performance was evaluated by regressing the network properties separately with respect to speeding up and IOI variability in ensemble playing. With increasing node strength, speeding up decreased $[\beta=-.015, \mathrm{SE}=.006, t=-2.61]$ and variability decreased $[\beta=-.009, \mathrm{SE}=.0016, t=-5.44]$, see Supplementary Figure S5. Interestingly, we also observed that TE tended to be higher in trials that deviated less from the instructed tempo, see Supplementary Figure S6.

\subsection{Variability in the mean-field model with discretely updated feedback}

The results of the modeling showed that the cycle duration variabilities of the mean-field hybrid Kuromoto model, adapted to have discretely updated feedback, reproduced the main experimental findings. The model results are summarized in Figure 4B and can be compared to the experimental results in Figure 4A. The adapted model reproduced the pattern of behavioural results at both the level of individual oscillators (relating to individuals in our drumming data) and the mean field (relating to our group-aggregate analyses). Specifically, for all group sizes, variability was higher for individual oscillators when playing in the group than when playing solo, but variability was always lowest for the mean field (group-aggregate). In addition, with increasing group size, the difference between individual variability and that of the mean field became greater, indicating increasing collective benefits with increasing group size. See Supplementary Materials, Figure S1B for a comparison with the null (pseudo-ensemble) condition, . A separate report will address the model's individual unit dynamics and co-variation in more detail (Delasanta et al., in preparation).

\section{Discussion}

The present study examined how group performance in a synchronization timing task depends on group size and interactions among group members. Our overall hypothesis was that the mean field would influence individuals in a group, thereby stabilizing group performance. We expected that larger groups would exhibit more stable performance due to a more stable mean field. This hypothesis was motivated by the collective dynamics that allow animals with limited capacity for interacting with each other to achieve mutual goals by exploiting processes such as positive collective feedback and wisdom of crowds (Sumpter, 2006). As expected, we observed that temporal variability in group drumming decreased with group size. This occurred only at the level of the ensemble or mean field, when the group was taken as a single entity. The effect was reversed for individual participants: their variability increased from solo to group playing conditions and with group size. We showed that this result agrees 
with a collective dynamics framework based on an adapted Kuramoto model. The model is consistent with the idea from theoretical biology that self-organizing systems can successfully coordinate with an external constraint by reducing their disorder at the macro-level at the expense of increasing their micro-level disorder (Kugler \& Turvey, 1987; Prokopenko, Polani, \& Ay, 2014) and that medium-sized groups exhibit commonalities with principles of organization found both in two-unit and large collectives (Kelso, 2021; Zhang et al., 2019).

The continuous-discrete Kuramoto system introduced here included fluctuating intrinsic frequencies and event-based feedback. Consistent with the discrete nature of time asynchronies in a drumming task, the model used a pulse function instead of continuous coupling. The implications of this idea reach beyond the topic of the present study. The combination of continuous movement and discrete sensory sampling of the environment is a frequently observed scenario; in the task of the present study, participants needed to take the discrete auditory feedback of the drum taps to inform their continuous movements to needed to create future drum taps. Although unexplored in the present study, group drumming can involve both continuous and discrete feedback (i.e., visual information about continuous arm, hand, and drumstick movements relates to the timing of individual drumming sounds). Future studies should address whether visual feedback during group drumming is informative, as well as explore how that visual information is captured within the individual and collective dynamics of the system.

Interestingly, in the octet group, individual variability was similar in both the synchronization-only condition, where the metronome pacing stimulus was present, and in the self-paced continuation stage of the SCT trials, where no pacing stimulus was present. This result implies that, although tempo accuracy and drift were superior when the pacing stimulus was present, the stability of group timing in a group of 8 participants may hit a ceiling, where performance becomes as stable as with a pacing stimulus.

Individuals' tempos tended to increase in solo trials and, as expected, this effect was amplified (i.e., speeding up was greater) when trying to stay synchronized with other participants (Okano, Shinya, \& Kudo, 2017). Surprisingly, the amount of speeding up was not higher for larger groups of drummers and it was even reduced for larger groups, at least at faster tempos. In contrast, accounts based on mutual prediction predict more speeding up for larger groups (Thomson, Murphy, \& Lukeman, 2018; Wolf, Vesper, Sebanz, Keller, \& Knoblich, 2019). This discrepancy highlights the importance of testing tempo drift with specific task constraints and avoiding hasty generalizations from smaller to larger 


\section{COLLECTIVE DYNAMICS IN GROUP DRUMMING}

groups. Indeed, tempo can also slow down in some circumstances. This was observed, for example, in a group synchronization task when participants synchronized their oscillations using swung pendulums, a task involving continuous visual coupling rather than discrete acoustic event coupling (Bardy et al., 2020).

Having summarized task performance in terms of consistency and accuracy, we next considered drumming dynamics. Individuals' autocorrelations exhibited negative coefficient peaks at odd lags and positive coefficient peaks at even lags. This is the typical error-correction process demonstrated when individuals synchronize their tapping with a stimulus or with each other in pairs (Konvalinka, Vuust, Roepstorff, \& Frith, 2010; Repp, 2005). With increasing group size, however, the magnitudes of the autocorrelation coefficient fluctuations with lag decreased; individuals' alternating patterns became smoother. This suggests that as group size increases drumming dynamics are dominated less by tap-bytap error-correction and more by a group dynamic. Interestingly, the group-aggregate onsets exhibited the same autocorrelation pattern as individuals, even in octets. This implies that the collective mean field behaviour spontaneously acquired some of the dynamic properties typically associated with individual performance.

Inter-personal coordination in duets exhibited the negative cross-correlation at lag0 and positive cross-correlation at lag1 expected from past studies (Repp, 2005). Yet, the coefficient magnitudes decreased with group size and all but disappeared for groups of 8, suggestive of a leader-less group coordination scenario (Heggli et al., 2019). In a small number of trials, we even observed positive lag0 cross-correlations in groups of 8 , a signature of anticipation emerging from dynamic interaction. This result is consistent with the idea that group members coordinated less with each other and more with the mean field. Along with a more slowly decaying auto-correlation function, this suggests the emergence of a larger-scale group dynamic in the octet. As we hypothesized, mutual prediction is not necessarily the only basis for coordination in larger groups. Note that there is no reason to treat collective dynamics and dyadic mutual prediction as mutually exclusive theoretical alternatives, only that collective dynamics is a mode of social interaction that emerges under certain constraints such as the number of interacting agents.

Characterizing inter-personal interaction on the basis of bivariate measures is typical in the context of dyadic paradigms. Larger groups, however, create the possibility for higher-order interactions. Network analysis was applied to the connectivity graph of transfer entropies among drummers. Larger groups had lower causal density but higher node strength. This is possible because individual nodes in 
larger groups project to a larger number of other individual nodes and suggests that in larger groups each individual has a decreased influence on the group, both less potential to destabilize the group and lower requirements to keep the group stable. We also found that higher node strength was associated with better overall performance, both in terms of variability and tempo accuracy.

Group timing tasks address some of the foundations of human social interaction. This area of research has been labeled the "dark matter" of social neuroscience (Dumas, de Guzman, Tognoli, \& Kelso, 2014; Insel, 2010; Schilbach et al., 2013) because it has not received enough attention despite the fact that large parts of the human cortex respond to social stimuli (Frith \& Frith, 2001). The temporal regularities in music make it an ideal stimulus to promote synchronous and cooperative movement, providing evidence as to why music is often present at emotional social gatherings such as weddings, funerals, parties, and political rallies. Feelings of affiliation and cooperation increase between people who have experienced synchronous movement with each other, even during infancy (Cirelli et al., 2014; Cirelli, Wan, \& Trainor, 2016; Hove \& Risen, 2009; Mogan, Fischer, \& Bulbulia, 2017; Trainor \& Cirelli, 2015; Valdesolo, Ouyang, \& DeSteno, 2010). The ability of music to strengthen social bonds within a group may have evolutionary roots that enhanced survival capabilities of the group (Huron, 2001; Savage et al., 2020; Trainor, 2015).

Participants in group activities sometimes report a so-called state of flow characterized by absorption, loss of sense of control, loss of self-consciousness, togetherness, and effortless action towards a shared goal (Csikszentmihalyi, 1990; Gaggioli, Chirico, Mazzoni, Milani, \& Riva, 2017; Hart, Noy, Feniger-Schaal, Mayo, \& Alon, 2014). Little is known about the underlying group processes that enable such experiences. Some of the required conditions have been studied in the context of group musical performance (Bishop, Cancino-Chacón, \& Goebl, 2019; D’Amario, Daffern, \& Bailes, 2018). Collective intentionality is often seen as an extrapolation of single-person cognitive processes in that individuals predict other individuals' goals and actions (Tomasello, 1999, 2014). Yet, the neural and cognitive mechanisms historically identified in single-person paradigms do not always map onto the ontology of social behavior (Przyrembel, Smallwood, Pauen, \& Singer, 2012). Collective intentionality can stand for an independent mode of social cognition (Satne \& Salice, 2018; Zahavi \& Satne, 2015).

Social cognition theory can even be turned upside-down by observing cases where individual agency arises from social interaction (de Jaegher \& Froese, 2009; Froese, Iizuka, \& Ikegami, 2014). The present work shows how collective dynamics can absorb individual action without every individual necessarily having to interact with and predict every other individual in the group. 


\section{COLLECTIVE DYNAMICS IN GROUP DRUMMING 27}

Funding. This research was supported by grants to LJT from the Social Science and Humanities

Research Council of Canada, the Natural Sciences and Engineering Research Council of Canada, the Canadian Institutes of Health Research, and the Canadian Institute for Advanced Research.

Acknowledgments. We would like to thank Daniel Bosnyak, Sally Stafford, Susan Marsh-Rollo and LIVELab staff. 


\section{COLLECTIVE DYNAMICS IN GROUP DRUMMING 28}

\section{References}

Alderisio, F., Bardy, B. G., \& di Bernardo, M. (2015). Entrainment and Synchronization in Heterogeneous Networks of Haken-Kelso-Bunz (HKB) Oscillators, 1-34. Retrieved from http://arxiv.org/abs/1509.00753

Bardy, B. G., Calabrese, C., De Lellis, P., Bourgeaud, S., Colomer, C., Pla, S., \& di Bernardo, M. (2020). Moving in unison after perceptual interruption. Scientific Reports, 10(1), 1-13. https://doi.org/10.1038/s41598-020-74914-z

Barrat, A., Barthélemy, M., Pastor-Satorras, R., \& Vespignani, A. (2004). The architecture of complex weighted networks. Proceedings of the National Academy of Sciences of the United States of America, 101(11). https://doi.org/10.1073/pnas.0400087101

Bates, D., Mächler, M., Bolker, B., \& Walker, S. (2014). Fitting linear mixed-effects models using lme4. ArXiv, 1406.5823.

Berdahl, A. M., Kao, A. B., Flack, A., Westley, P. A. H., Codling, E. A., Couzin, I. D., ... Biro, D. (2018). Collective animal navigation and migratory culture: From theoretical models to empirical evidence. Philosophical Transactions of the Royal Society B: Biological Sciences, 373(1746). https://doi.org/10.1098/rstb.2017.0009

Bishop, L., Cancino-Chacón, C., \& Goebl, W. (2019). Moving to Communicate, Moving to Interact. Music Perception, 37(1). https://doi.org/10.1525/mp.2019.37.1.1

Breakspear, M., Heitmann, S., \& Daffertshofer, A. (2010). Generative models of cortical oscillations: Neurobiological implications of the Kuramoto model. Frontiers in Human Neuroscience, 4(November), 190. https://doi.org/10.3389/fnhum.2010.00190

Brown, J., Bossomaier, T., \& Barnett, L. (2020). Information flow in finite flocks. Scientific Reports, 10(1). https://doi.org/10.1038/s41598-020-59080-6

Chang, A., Kragness, H. E., Livingstone, S. R., Bosnyak, D. J., \& Trainor, L. J. (2019). Body sway reflects joint emotional expression in music ensemble performance. Scientific Reports, 9(1), 1-11. https://doi.org/10.1038/s41598-018-36358-4

Chang, A., Livingstone, S. R., Bosnyak, D. J., \& Trainor, L. J. (2017). Body sway reflects leadership in joint music performance. Proceedings of the National Academy of Sciences. https://doi.org/10.1073/pnas.1617657114

Chauvigné, L. A. S., Walton, A., Richardson, M. J., \& Brown, S. (2019). Multi-person and multisensory synchronization during group dancing. Human Movement Science, 63, 199-208. https://doi.org/10.1016/j.humov.2018.12.005

Cirelli, L. K., Einarson, K. M., \& Trainor, L. J. (2014). Interpersonal synchrony increases prosocial behavior in infants. Developmental Science, 17(6), 1003-1011. https://doi.org/10.1111/desc.12193

Cirelli, L. K., Trehub, S. E., \& Trainor, L. J. (2018). Rhythm and melody as social signals for infants. Annals of the New York Academy of Sciences, 1423(1), 66-72. https://doi.org/10.1111/nyas.13580

Cirelli, L. K., Wan, S. J., \& Trainor, L. J. (2016). Social Effects of Movement Synchrony: Increased Infant Helpfulness only Transfers to Affiliates of Synchronously Moving Partners. Infancy, 21(6), 807-821. https://doi.org/10.1111/infa.12140 
Conard, N. J., Malina, M., \& Münzel, S. C. (2009). New flutes document the earliest musical tradition in southwestern Germany. Nature, 460(7256), 737. https://doi.org/10.1038/nature08169

Couzin, I. D. (2018). Synchronization: The Key to Effective Communication in Animal Collectives. Trends in Cognitive Sciences. https://doi.org/10.1016/j.tics.2018.08.001

Csardi, G., \& Nepusz, T. (2006). The igraph software package for complex network research. InterJournal Complex Systems, Complex Sy(1695).

Csikszentmihalyi, M. (1990). Flow: The psychology of optimal experience. New York, NY: Harper \& Row.

D’Amario, S., Daffern, H., \& Bailes, F. (2018). Synchronization in singing duo performances: The roles of visual contact and leadership instruction. Frontiers in Psychology, 9(JUL). https://doi.org/10.3389/fpsyg.2018.01208

Dayan, P., \& Abbott, L. F. (2001). Theoretical Neuroscience: Computational and Mathematical Modeling of Neural Systems. Computational and Mathematical Modeling of Neural ....

de Jaegher, H., \& Froese, T. (2009). On the role of social interaction in individual agency. Adaptive Behavior, 17(5). https://doi.org/10.1177/1059712309343822

Dean, R. T., \& Dunsmuir, W. T. M. (2016). Dangers and uses of cross-correlation in analyzing time series in perception, performance, movement, and neuroscience: The importance of constructing transfer function autoregressive models. Behavior Research Methods, 48(2), 783-802. https://doi.org/10.3758/s13428-015-0611-2

Dotov, D., Cochen de Cock, V., Geny, C., Ihalainen, P., Moens, B., Leman, M., ... Dalla Bella, S. (2019). The role of interaction and predictability in the spontaneous entrainment of movement. Journal of Experimental Psychology: General, 148(6), 1041-1057. https://doi.org/10.1037/xge0000609

Dotov, D., \& Froese, T. (2018). Entraining chaotic dynamics: A novel movement sonification paradigm could promote generalization. Human Movement Science, 61. https://doi.org/10.1016/j.humov.2018.06.016

Dumas, G., de Guzman, G. C., Tognoli, E., \& Kelso, J. A. S. (2014). The human dynamic clamp as a paradigm for social interaction. Proceedings of the National Academy of Sciences of the United States of America, 111(35), E3726-34. https://doi.org/10.1073/pnas.1407486111

Farine, D. R., Strandburg-Peshkin, A., Couzin, I. D., Berger-Wolf, T. Y., \& Crofoot, M. C. (2017). Individual variation in local interaction rules can explain emergent patterns of spatial organisation in wild baboons. Proceedings of the Royal Society B: Biological Sciences, 284, 20162243. https://doi.org/10.1098/rspb.2016.2243

Frank, T. D., Daffertshofer, A., Peper, C. E., Beek, P. J., \& Haken, H. (2000). Towards a comprehensive theory of brain activity: Physica D: Nonlinear Phenomena, 144(1-2), 62-86. https://doi.org/10.1016/S0167-2789(00)00071-3

Friston, K., \& Frith, C. (2015). A Duet for one. Consciousness and Cognition, 36, 390-405. https://doi.org/10.1016/j.concog.2014.12.003

Frith, U., \& Frith, C. (2001). The Biological Basis of Social Interaction. Current Directions in Psychological Science, 10(5), 151-155. https://doi.org/10.1111/1467-8721.00137 


\section{COLLECTIVE DYNAMICS IN GROUP DRUMMING}

Froese, T., Iizuka, H., \& Ikegami, T. (2014). Embodied social interaction constitutes social cognition in Pairs of humans: A minimalist virtual reality experiment. Scientific Reports, 4. https://doi.org/10.1038/srep03672

Gaggioli, A., Chirico, A., Mazzoni, E., Milani, L., \& Riva, G. (2017). Networked Flow in musical bands. Psychology of Music, 45(2). https://doi.org/10.1177/0305735616665003

Galton, F. (1907). Vox Populi ( The Wisdom of Crowds ). Nature, 75.

Gamba, M., Torti, V., Estienne, V., Randrianarison, R. M., Valente, D., Rovara, P., ... Giacoma, C. (2016). The Indris have got rhythm! Timing and pitch variation of a primate song examined between sexes and age classes. Frontiers in Neuroscience, 10(JUN), 249. https://doi.org/10.3389/fnins.2016.00249

Goldstone, R. L., \& Roberts, M. E. (2006). Self-organized trail systems in groups of humans. Complexity, 11(6). https://doi.org/10.1002/cplx.20135

Gourévitch, B., \& Eggermont, J. J. (2007). Evaluating information transfer between auditory cortical neurons. Journal of Neurophysiology, 97(3), 2533-2543. https://doi.org/10.1152/jn.01106.2006

Greenfield, M. D., Marin-Cudraz, T., \& Party, V. (2017). Evolution of synchronies in insect choruses. Biological Journal of the Linnean Society, 122(3), 487-504.

https://doi.org/10.1093/biolinnean/blx096

Hart, Y., Noy, L., Feniger-Schaal, R., Mayo, A. E., \& Alon, U. (2014). Individuality and togetherness in joint improvised motion. PLOS ONE, 9(2). https://doi.org/10.1371/journal.pone.0087213

Heggli, O. A., Cabral, J., Konvalinka, I., Vuust, P., \& Kringelbach, M. L. (2019). A Kuramoto model of self-other integration across interpersonal synchronization strategies. PLOS Computational Biology, 15(10), e1007422. https://doi.org/10.1371/journal.pcbi.1007422

Heggli, O. A., Konvalinka, I., Kringelbach, M. L., \& Vuust, P. (2021). A Metastable Attractor Model of Self-Other Integration (MEAMSO) in Rhythmic Synchronization. Philosophical Transactions of the Royal Society B: Biological Sciences.

Honing, H., ten Cate, C., Peretz, I., \& Trehub, S. E. (2015). Without it no music: cognition, biology and evolution of musicality. Philosophical Transactions of the Royal Society B: Biological Sciences, 370(1664), 20140088. https://doi.org/10.1098/rstb.2014.0088

Hove, M. J., \& Risen, J. L. (2009). It’s All in the Timing: Interpersonal Synchrony Increases Affiliation. Social Cognition, 27(6), 949-960. https://doi.org/10.1521/soco.2009.27.6.949

Huron, D. (2001). Is music an evolutionary adaptation? In Annals of the New York Academy of Sciences (Vol. 930). https://doi.org/10.1111/j.1749-6632.2001.tb05724.X

Insel, T. R. (2010). The Challenge of Translation in Social Neuroscience: A Review of Oxytocin, Vasopressin, and Affiliative Behavior. Neuron. https://doi.org/10.1016/j.neuron.2010.03.005

Ispolatov, Y. (2015). Computing in fish schools. ELife, 5(e12852). https://doi.org/10.7554/eLife.12852

Ito, S., Hansen, M. E., Heiland, R., Lumsdaine, A., Litke, A. M., \& Beggs, J. M. (2011). Extending Transfer Entropy Improves Identification of Effective Connectivity in a Spiking Cortical Network Model. PLoS ONE, 6(11), e27431. https://doi.org/10.1371/journal.pone.0027431

Jacobs, P. A., Lengauer, C. C., Bruschi, C. V, Garre, M., Gjuracic, K., \& Mccoy, E. E. (2007). From 


\section{COLLECTIVE DYNAMICS IN GROUP DRUMMING}

disorder to order in marching locusts. Science, 317(August), 490-494.

https://doi.org/10.5061/dryad.5t110.Supplementary

Keller, P. E., Novembre, G., \& Hove, M. J. (2014). Rhythm in joint action: psychological and neurophysiological mechanisms for real-time interpersonal coordination. Philosophical

Transactions of the Royal Society of London. Series B, Biological Sciences, 369(1658), 20130394. https://doi.org/10.1098/rstb.2013.0394

Kelso, J. A. S. (2021). Unifying large- and small-scale theories of coordination. Entropy, 23(5), 537. https://doi.org/10.3390/e23050537

Konvalinka, I., Vuust, P., Roepstorff, A., \& Frith, C. D. (2010). Follow you, follow me: Continuous mutual prediction and adaptation in joint tapping. Quarterly Journal of Experimental Psychology, 63(11), 2220-2230. https://doi.org/10.1080/17470218.2010.497843

Kugler, P. N., \& Turvey, M. T. (1987). Information, natural law, and the self-assembly of rhythmic movement. Hillsdale, NJ: Lawrence Erlbaum Associates, Inc.

Kuramoto, Y. (1975). Self-entrainment of a population of coupled non-linear oscillators. In International Symposium on Mathematical Problems in Theoretical Physics (Vol. 39).

Kuznetsova, A., Brockhoff, P. B., \& Christensen, R. H. B. (2017). ImerTest Package: Tests in Linear Mixed Effects Models . Journal of Statistical Software, 82(13), 1-26. https://doi.org/10.18637/jss.v082.i13

Lungarella, M., Pegors, T., Bulwinkle, D., \& Sporns, O. (2005). Methods for quantifying the informational structure of sensory and motor data. In Neuroinformatics (Vol. 3). https://doi.org/10.1385/NI:3:3:243

Lungarella, M., \& Sporns, O. (2006). Mapping information flow in sensorimotor networks. PLoS Computational Biology, 2(10). https://doi.org/10.1371/journal.pcbi.0020144

Madison, G., Karampela, O., Ullén, F., \& Holm, L. (2013). Effects of practice on variability in an isochronous serial interval production task: Asymptotical levels of tapping variability after training are similar to those of musicians. Acta Psychologica, 143(1), 119-128. https://doi.org/10.1016/j.actpsy.2013.02.010

Mann, R. P., Faria, J., Sumpter, D. J. T., \& Krause, J. (2013). The dynamics of audience applause. Journal of the Royal Society Interface, 10(85), 20130466. https://doi.org/10.1098/rsif.2013.0466

Manning, F. C., Harris, J., \& Schutz, M. (2017). Temporal prediction abilities are mediated by motor effector and rhythmic expertise. Experimental Brain Research, 235(3), 861-871. https://doi.org/10.1007/s00221-016-4845-8

Miller, N., Garnier, S., Hartnett, A. T., \& Couzin, I. D. (2013). Both information and social cohesion determine collective decisions in animal groups. Proceedings of the National Academy of Sciences, 110(13), 5263-5268. https://doi.org/10.1073/pnas.1217513110

Mogan, R., Fischer, R., \& Bulbulia, J. A. (2017). To be in synchrony or not? A meta-analysis of synchrony's effects on behavior, perception, cognition and affect. Journal of Experimental Social Psychology, 72, 13-20. https://doi.org/10.1016/j.jesp.2017.03.009

Newman, M. E. J., Barabási, A. L., \& Watts, D. J. (2011). The structure and dynamics of networks. The Structure and Dynamics of Networks (Vol. 9781400841356). https://doi.org/10.1007/s10955-006- 


\section{7-8}

Noori, R., Park, D., Griffiths, J. D., Bells, S., Frankland, P. W., Mabbott, D., \& Lefebvre, J. (2020). Activity-dependent myelination: A glial mechanism of oscillatory self-organization in large-scale brain networks. Proceedings of the National Academy of Sciences of the United States of America, 117(24), 13227-13237. https://doi.org/10.1073/pnas.1916646117

Okano, M., Shinya, M., \& Kudo, K. (2017). Paired Synchronous Rhythmic Finger Tapping without an External Timing Cue Shows Greater Speed Increases Relative to Those for Solo Tapping. Scientific Reports. https://doi.org/10.1038/srep43987

Ouiller, O., de Guzman, G., Jantzen, K., Lagarde, J., \& Kelso, J. (2008). Social coordination dynamics: Measuring human bonding. Social Neuroscience, 3(2), 178-192.

Parrish, J. K., Viscido, S. V., \& Grünbaum, D. (2002). Self-organized fish schools: An examination of emergent properties. Biological Bulletin, 202(3), 296-305. https://doi.org/10.2307/1543482

Patel, A. D., \& Iversen, J. R. (2014). The evolutionary neuroscience of musical beat perception: The Action Simulation for Auditory Prediction (ASAP) hypothesis. Frontiers in Systems Neuroscience, 8(MAY). https://doi.org/10.3389/fnsys.2014.00057

Prokopenko, M., Polani, D., \& Ay, N. (2014). On the Cross-Disciplinary Nature of Guided SelfOrganisation (pp. 3-15). Springer, Berlin, Heidelberg. https://doi.org/10.1007/978-3-642-537349_1

Puckette, M. S. (1996). Pure Data. In Proceedings, International Computer Music Conference. San Francisco, CA.

Ravignani, A., Bowling, D., \& Fitch, W. T. (2014). Chorusing, synchrony and the evolutionary functions of rhythm. Frontiers in Psychology, 5(SEP), 1-15. https://doi.org/10.3389/fpsyg.2014.01118

Ravignani, A., Verga, L., \& Greenfield, M. D. (2019). Interactive rhythms across species: the evolutionary biology of animal chorusing and turn-taking. Annals of the New York Academy of Sciences, 1453(1), 12-21. https://doi.org/10.1111/nyas.14230

Repp, B. H. (2005). Sensorimotor synchronization: a review of the tapping literature. Psychonomic Bulletin \& Review, 12(6), 969-992. https://doi.org/10.3758/BF03206433

Rio, K. W., Dachner, G. C., \& Warren, W. H. (2018). Local interactions underlying collective motion in human crowds. Proceedings of the Royal Society B: Biological Sciences, 285(1878), 20180611. https://doi.org/10.1098/rspb.2018.0611

Roman, I. R., Washburn, A., Large, E. W., Chafe, C., \& Fujioka, T. (2019). Delayed feedback embedded in perception-action coordination cycles results in anticipation behavior during synchronized rhythmic action: A dynamical systems approach. PLoS Computational Biology, 15(10). https://doi.org/10.1371/journal.pcbi.1007371

Rosenthal, S. B., Twomey, C. R., Hartnett, A. T., Wu, H. S., \& Couzin, I. D. (2015). Revealing the hidden networks of interaction in mobile animal groups allows prediction of complex behavioral contagion. Proceedings of the National Academy of Sciences, 112(15), 4690-4695. https://doi.org/10.1073/pnas.1420068112

Salimpoor, V. N., Benovoy, M., Larcher, K., Dagher, A., \& Zatorre, R. J. (2011). Anatomically distinct 


\section{COLLECTIVE DYNAMICS IN GROUP DRUMMING}

dopamine release during anticipation and experience of peak emotion to music. In Nature Neuroscience (Vol. 14). https://doi.org/10.1038/nn.2726

Satne, G., \& Salice, A. (2018). Shared intentionality and the cooperative evolutionary hypothesis. In A. Fiebich (Ed.), Minimal Cooperation and Shared Agency (Vol. 2018). Berlin, Germany: Springer.

Savage, P. E., Brown, S., Sakai, E., \& Currie, T. E. (2015). Statistical universals reveal the structures and functions of human music. Proceedings of the National Academy of Sciences of the United States of America, 112(29). https://doi.org/10.1073/pnas.1414495112

Savage, P. E., Loui, P., Tarr, B., Schachner, A., Glowacki, L., Mithen, S., \& Fitch, W. T. (2020). Music as a coevolved system for social bonding. Behavioral and Brain Sciences.

https://doi.org/10.1017/S0140525X20000333

Schilbach, L., Timmermans, B., Reddy, V., Costall, A., Bente, G., Schlicht, T., \& Vogeley, K. (2013, August 25). A second-person neuroscience in interaction. Behavioral and Brain Sciences.

Cambridge University Press. https://doi.org/10.1017/S0140525X12002452

Schmidt, R. C., Carello, C., \& Turvey, M. T. (1990). Phase Transitions and Critical Fluctuations in the Visual Coordination of Rhythmic Movements Between People. Journal of Experimental Psychology: Human Perception and Performance, 16(2), 227-247. https://doi.org/10.1037/00961523.16.2.227

Schmidt, R. C., \& O’Brien, B. (1997). Evaluating the Dynamics of Unintended Interpersonal Coordination. Ecological Psychology, 9(3), 189-206. https://doi.org/10.1207/s15326969eco0903_2

Schreiber, T. (2000). Measuring information transfer. Physical Review Letters, 85(2), 461-464. https://doi.org/10.1103/PhysRevLett.85.461

Seth, A. K., Barrett, A. B., \& Barnett, L. (2011). Causal density and integrated information as measures of conscious level. Philosophical Transactions of the Royal Society A: Mathematical, Physical and Engineering Sciences. https://doi.org/10.1098/rsta.2011.0079

Seth, A. K., \& Edelman, G. M. (2004). Environment and Behavior Influence the Complexity of Evolved Neural Networks. Adaptive Behavior, 12(1), 5-20. https://doi.org/10.1177/105971230401200103

Shahal, S., Wurzberg, A., Sibony, I., Duadi, H., Shniderman, E., Weymouth, D., ... Fridman, M. (2020). Synchronization of complex human networks. Nature Communications, 11(1). https://doi.org/10.1038/s41467-020-17540-7

Singer, J. D., \& Willett, J. B. (2003). Applied Longitudinal Data Analysis: Modeling Change and Event Occurrence. Oxford University Press.

Stoffregen, T. A., Villard, S., Kim, C. G., Ito, K., \& Bardy, B. G. (2009). Coupling of Head and Body Movement With Motion of the Audible Environment. Journal of Experimental Psychology: Human Perception and Performance, 35(4). https://doi.org/10.1037/a0014251

Strogatz, S. H. (2000). From Kuramoto to Crawford: exploring the onset of synchronization in populations of coupled oscillators. Physica D: Nonlinear Phenomena, 143, 1-20. https://doi.org/10.1016/S0167-2789(00)00094-4

Sumpter, D. J. T. (2006). The principles of collective animal behaviour. Philosophical Transactions of 
the Royal Society B: Biological Sciences, 361(1465), 5-22. https://doi.org/10.1098/rstb.2005.1733

Surowiecki, J. (2005). The Wisdom of Crowds. Anchor.

Thomson, M., Murphy, K., \& Lukeman, R. (2018). Groups clapping in unison undergo size-dependent error-induced frequency increase. Scientific Reports, 8(1). https://doi.org/10.1038/s41598-01718539-9

Tomasello, M. (1999). The cultural origins of human cognition. Cambridge, MA: Harvard University Press.

Tomasello, M. (2014). Natural History of Human Thinking. Cambridge, MA: Harvard University Press.

Torney, C., Neufeld, Z., \& Couzin, I. D. (2009). Context-dependent interaction leads to emergent search behavior in social aggregates. Proceedings of the National Academy of Sciences, 106(52), 22055-22060. https://doi.org/10.1073/pnas.0907929106

Trainor, L. J. (2015). The origins of music in auditory scene analysis and the roles of evolution and culture in musical creation. Philosophical Transactions of the Royal Society B: Biological Sciences, 370(1664). https://doi.org/10.1098/rstb.2014.0089

Trainor, L. J., \& Cirelli, L. (2015). Rhythm and interpersonal synchrony in early social development. Annals of the New York Academy of Sciences, 1337(1), 45-52. https://doi.org/10.1111/nyas.12649

Trainor, L. J., \& Marsh-Rollo, S. (2019). Rhythm, Meter, and Timing: The Heartbeat of Musical Development. In The Oxford Handbook of Music and the Brain (pp. 591-622). https://doi.org/10.1093/oxfordhb/9780198804123.013.24

Valdesolo, P., Ouyang, J., \& DeSteno, D. (2010). The rhythm of joint action: Synchrony promotes cooperative ability. Journal of Experimental Social Psychology, 46(4), 693-695. https://doi.org/10.1016/J.JESP.2010.03.004

van der Steen, M. C. (Marieke), \& Keller, P. E. (2013). The ADaptation and Anticipation Model (ADAM) of sensorimotor synchronization. Frontiers in Human Neuroscience, 7, 253. https://doi.org/10.3389/fnhum.2013.00253

Vilar, L., Araújo, D., Davids, K., \& Bar-Yam, Y. (2013). Science of winning soccer: Emergent patternforming dynamics in association football. Journal of Systems Science and Complexity, 26(1), 7384. https://doi.org/10.1007/s11424-013-2286-z

Warren, W. H. (2018, July 11). Collective Motion in Human Crowds. Current Directions in Psychological Science, p. 096372141774674. https://doi.org/10.1177/0963721417746743

Wiener, N. (1956). The Theory of Prediction. In Modern Mathematics for Engineers (Vol. 58, pp. 323329). McGraw-Hill. Retrieved from https://ci.nii.ac.jp/naid/10010977018

Wolf, T., Vesper, C., Sebanz, N., Keller, P. E., \& Knoblich, G. (2019). Combining Phase Advancement and Period Correction Explains Rushing during Joint Rhythmic Activities. Scientific Reports, 9(1), 9350. https://doi.org/10.1038/s41598-019-45601-5

Wolpert, D. M., Doya, K., \& Kawato, M. (2003). A unifying computational framework for motor control and social interaction. Philosophical Transactions of the Royal Society B: Biological Sciences. https://doi.org/10.1098/rstb.2002.1238

Zahavi, D., \& Satne, G. (2015). Varieties of shared intentionality: Tomasello and classical 


\section{COLLECTIVE DYNAMICS IN GROUP DRUMMING 35}

phenomenology. In Beyond the Analytic-Continental Divide: Pluralist Philosophy in the TwentyFirst Century. https://doi.org/10.4324/9781315766621

Zentner, M., \& Eerola, T. (2010). Rhythmic engagement with music in infancy. PNAS, 107(13), 57685773. https://doi.org/10.1073/pnas.1000121107

Zhang, M., Beetle, C., Kelso, J. A. S., \& Tognoli, E. (2019). Connecting empirical phenomena and theoretical models of biological coordination across scales. Journal of The Royal Society Interface, 16(157), 20190360. https://doi.org/10.1098/rsif.2019.0360 